\title{
Limit theorems for pure death processes coming down from infinity
}

\author{
Serik Sagitov* and Thibaut France ${ }^{\dagger}$
}

November 14, 2018

\begin{abstract}
We consider a pure death process $(Z(t), t \geq 0)$ with death rates $\lambda_{n}$ satisfying the condition $\sum_{n=2}^{\infty} \lambda_{n}^{-1}<\infty$ of coming from infinity, $Z(0)=$ $\infty$, down to an absorbing state $n=1$. We establish limit theorems for $Z(t)$ as $t \rightarrow 0$, which strengthen the results that can be extracted from [1. We also prove a large deviation theorem assuming that $\lambda_{n}$ regularly vary as $n \rightarrow \infty$ with an index $\beta>1$. It generalises a similar statement with $\beta=2$ obtained in [4] for $\lambda_{n}=\left(\begin{array}{l}n \\ 2\end{array}\right)$.
\end{abstract}

Key words: Almost sure convergence, large deviations, Kingman's coalescent.

\section{Introduction}

The number of lineages in the Kingman coalescent [5] instantaneously comes down from infinity by jumps $n \rightarrow n-1$ at rate $\lambda_{n}=\left(\begin{array}{c}n \\ 2\end{array}\right)$. As a natural extention of the Kingman setting, we consider a pure death process $(Z(t), t \geq 0)$ with an absorbing state $n=1$, described by a sequence of death rates $\left(\lambda_{n}, n \geq 2\right)$ such that

$$
\sum_{n=2}^{\infty} \lambda_{n}^{-1}<\infty
$$

Assume that $Z(0)=\infty$ and denote by $T_{n}$ the first time when $Z(t)$ hits a given state $n \geq 1$. Clearly,

$$
T_{n}=X_{n+1}+X_{n+2}+\ldots,
$$

where $X_{2}, X_{3}, \ldots$ are independent exponentially distributed holding times with $\mathbb{E} X_{i}=\lambda_{i}^{-1}$. Under condition (1), the mean value of the hitting time $T_{n}$

$$
A_{n}=\mathbb{E} T_{n}=\sum_{i=n+1}^{\infty} \lambda_{i}^{-1}
$$

${ }^{*}$ Chalmers University and University of Gothenburg, 41296 Gothenburg, Sweden. Email address: serik@chalmers.se

†École Polytechnique, route de Saclay, 91128 Palaiseau Cedex-France; Email address: thibaut.france@polytechnique.edu 
is finite, and $A_{n} \rightarrow 0$ as $n \rightarrow \infty$. Thus the process instantaneously comes down from infinity, in that $\mathbb{P}(Z(t)=\infty \mid Z(0)=\infty)=0$ for any $t>0$.

In this paper we are interested in the asymptotic properties of $Z(t)$ as $t \rightarrow 0$. In view of the relation $\{Z(t)>n\}=\left\{T_{n}>t\right\}$, the step function

$$
v(t)=\sum_{n=2}^{\infty} n 1_{\left[A_{n}, A_{n-1}\right)}(t)+1_{\left[A_{1}, \infty\right)}(t),
$$

being a generalised inverse of the sequence $\left(A_{n}\right)$, gives the speed of coming down from infinity for the process $Z(t)$, cf [2]. Recall that for the Kingman coalescent, $A_{n}=2 /(n+1)$ and $v(t) \sim 2 / t$ as $t \rightarrow 0$.

Our main results are presented in Sections 2 and 4 . Section 2 contains two comprehensive limit theorems. Theorem 11 dealing with $T_{n}$, can be deduced from more general results recently obtained in [1] for birth-death processes, however, our specialised proofs are more direct. Theorem 2 dealing with $Z(t)$, improves the conditions for the laws of large numbers and the central limit theorem compared to their counterparts given in [1. In particular, our Theorem 2 (i) states that $Z(t) / v(t) \rightarrow 1$ in probability as $t \rightarrow 0$ under a very mild restriction

$$
\limsup _{n \rightarrow \infty} \frac{A_{n x}}{A_{n}}<1, \quad \text { for all } x>1 .
$$

Notational agreement: whenever in place of an integer index we put a non-integer number, say $u$, we mean that the actual index is $\lfloor u\rfloor$, so that $A_{n x}:=A_{\lfloor n x\rfloor}$.

In Section 3 we give a number of examples illustrating a wide range of possible growth patterns covered by Theorem 2 for the speed function $v(t)$ as $t \rightarrow 0$. Section 4 presents an explicit large deviation theorem generalizing a recent result in [4] obtained for the Kingman coalescent. The remaining sections are devoted to self-contained proofs.

Notice that our results can be also interpreted in terms of an explosive pure birth process $N(u)=Z\left(T_{1}-u\right)$ obtained from the pure death process $\left(Z(t), 0<t \leq T_{1}\right)$ by time reversing. The time-reversed process $N(u)$ can be viewed as a simple model for the number of neutrons at time $u$ in a nuclear chain reaction exploding at the finite random time $T_{1}$, see [7] and [9. Knowing the speed of explosion $v(t)$ and the current population size $N(u)$, one can hope to predict the time $t=T_{1}-u$ left to the explosion event, cf [8].

\section{Limit theorems for $T_{n}$ and $Z(t)$}

Recall (2) and put

$$
B_{n}^{2}=\operatorname{Var}\left(T_{n}\right)=\sum_{i=n+1}^{\infty} \lambda_{i}^{-2}, \quad C_{n}^{3}=\sum_{i=n+1}^{\infty} \lambda_{i}^{-3} .
$$


Theorem 1. Consider a pure death process with parameters $\left(\lambda_{n}\right)$ satisfying condition (1).

(i) If

$$
\lambda_{n} / \lambda_{n+1} \rightarrow \alpha \in[0,1), \quad n \rightarrow \infty,
$$

then for each fixed $x \geq 0$,

$$
\mathbb{P}\left(A_{n}^{-1} T_{n} \leq x\right) \rightarrow F_{\alpha}(x), \quad n \rightarrow \infty,
$$

where the limit distribution has Laplace transform

$$
\int_{0}^{\infty} e^{-u x} d F_{\alpha}(x)=\prod_{i \geq 0} \frac{1}{u \alpha^{i}(1-\alpha)+1} .
$$

(ii) $A_{n}^{-1} T_{n} \rightarrow 1$ in probability as $n \rightarrow \infty$, if

$$
\lambda_{n}^{-1}=o\left(A_{n}\right), \quad n \rightarrow \infty .
$$

(iii) $A_{n}^{-1} T_{n} \rightarrow 1$ almost surely as $n \rightarrow \infty$, if

$$
\sum_{i=1}^{\infty}\left(\lambda_{i+1} A_{i}\right)^{-2}<\infty
$$

(iv) If (5) holds and furthermore

$$
C_{n}=o\left(B_{n}\right), \quad n \rightarrow \infty,
$$

then for all $x \in(-\infty, \infty)$,

$$
\mathbb{P}\left(\frac{T_{n}-A_{n}}{B_{n}} \leq x\right) \rightarrow \Phi(x), \quad n \rightarrow \infty,
$$

where $\Phi(x)$ is the standard normal distribution function.

\section{Remarks 1-5}

1. Condition (4) implies

$$
\lambda_{n+1} A_{n} \rightarrow 1+\alpha+\alpha^{2}+\ldots=\frac{1}{1-\alpha},
$$

yielding

$$
\left(\lambda_{n+i} A_{n}\right)^{-1} \rightarrow \alpha^{i-1}(1-\alpha), \quad i \geq 1 .
$$

It is equivalent to the condition

$$
\left(\lambda_{n+1} A_{n}\right)^{-1} \rightarrow \tilde{\alpha}:=1-\alpha \in[0,1),
$$

which in [1] is used to define the "fast regime" of coming down from infinity. The reverse part of this equivalence is seen from the recursion

$$
\lambda_{n+1} A_{n}=1+\frac{\lambda_{n+1}}{\lambda_{n+2}}\left(\lambda_{n+2} A_{n+1}\right) .
$$


2. Condition (4) implies $A_{n+1} / A_{n} \rightarrow \alpha$, and therefore,

$$
A_{n x}=o\left(A_{n}\right), \quad \text { for all } x>1 .
$$

3. Condition (1) together with

$$
\lambda_{n} / \lambda_{n+1} \rightarrow 1, \quad n \rightarrow \infty
$$

imply condition (5).

4. Condition (5) is equivalent to

$$
B_{n}=o\left(A_{n}\right), \quad n \rightarrow \infty .
$$

To verify this, let us fix an arbitrary $\epsilon \in(0,1)$. If 111$)$ holds, then for sufficiently large $n$,

$$
\lambda_{n+1}^{-2} \leq B_{n}^{2} \leq \epsilon^{2} A_{n}^{2},
$$

so that $\lambda_{n+1}^{-1} \leq \epsilon\left(\lambda_{n+1}^{-1}+A_{n+1}\right)$ and $\lambda_{n+1}^{-1} \leq \epsilon(1-\epsilon)^{-1} A_{n+1}$, which implies (5). On the other hand, given (5),

$$
A_{n}^{2}-B_{n}^{2}=2 \sum_{i=n+1}^{\infty} \sum_{j=i+1}^{\infty} \lambda_{i}^{-1} \lambda_{j}^{-1}=2 \sum_{i=n+1}^{\infty} \lambda_{i}^{-1} A_{i} \geq \epsilon^{-1} B_{n}^{2}
$$

for all sufficiently large $n$, which yields 111.

5. Condition (6) implies (11) due to the inequality

$$
A_{n}^{2} \sum_{i=n}^{\infty}\left(\lambda_{i+1} A_{i}\right)^{-2} \geq B_{n}^{2}
$$

Theorem 2. Consider a pure death process with parameters $\left(\lambda_{n}\right)$ satisfying conditions (1) and (3).

(i) $Z(t) / v(t) \rightarrow 1$ in probability as $t \rightarrow 0$.

(ii) $Z(t) / v(t) \rightarrow 1$ almost surely, if for each $\epsilon \in(0,1)$,

$$
\sum_{i=1}^{\infty}\left(\lambda_{i+1} A_{i(1-\epsilon)}\right)^{-2}<\infty .
$$

(iii) If condition (4) holds, then for each $k=0, \pm 1, \pm 2, \ldots$,

$$
\mathbb{P}\left(Z\left(A_{n}\right) \leq n+k\right) \rightarrow F_{\alpha}\left(\alpha^{-k}\right), \quad n \rightarrow \infty .
$$

(iv) Let (5) and (7) hold. If $b_{n}=o(n)$ is such that for all $x \in(-\infty, \infty)$,

$$
\frac{A_{n}-A_{n+x b_{n}}}{B_{n+x b_{n}}} \rightarrow h(x), \quad n \rightarrow \infty,
$$

then

$$
\mathbb{P}\left(\frac{Z(t)-v(t)}{b_{v(t)}} \leq x\right) \rightarrow \Phi(h(x)), \quad t \rightarrow 0
$$


An important class of the pure death processes coming down from infinity is set out by the constraint

$$
\lambda_{n}=n^{\beta} L(n), \quad \beta>1,
$$

where the function $L:[1, \infty) \rightarrow(0, \infty)$ is assumed to slowly vary at infinity. For the Kingman coalescent, this condition holds with $\beta=2$. By the properties of regularly varying functions, see [3], condition 13 entails

$$
A_{n}=n^{1-\beta} L_{1}(n), \quad L_{1}(n) \sim(\beta-1)^{-1} L^{-1}(n), \quad n \rightarrow \infty,
$$

implying that $v(t)$ regularly varies at zero with index $\frac{1}{1-\beta}$. In this case, condition (3) holds but not (9). The following statement is easily obtained from parts (ii) and (iv) of Theorem 2 .

Corollary 3. If condition (13) holds, then $Z(t) / v(t) \rightarrow 1$ almost surely and the limit distribution of $\frac{Z(t)-v(t)}{\sqrt{v(t)}}$ is normal with mean zero and variance $\frac{1}{2 \beta-1}$.

\section{Remarks 6-9}

6. Parts (i) and (ii) of Theorem 2 should be compared to the pure death case of Theorems 4.3 and 4.4 in [1. Our laws of large numbers are stated under much weaker conditions. Notice that (6) implies (12).

7. Part (iii) has no counterpart in [1].

8. Part (iv) should be compared to the pure death case of Proposition 4.6 in [1.

9. Corollary 3 should be compared to Theorem 5.1 in [1].

\section{Examples}

Below we give five simple examples illustrating the wide range of regimes covered by Theorem 2. For all our examples, the key condition (1) is easily verified. Paradoxically, the faster is the decay of $A_{n}$ as $n \rightarrow \infty$, the slower is the speed of coming down from infinity.

1. Let $A_{n}=(\log n)^{-a}$ for some $a>0$. Then, as $n \rightarrow \infty$,

$\lambda_{n} \sim a^{-1} n(\log n)^{1+a}, \quad B_{n} \sim a^{-1} n^{-1 / 2}(\log n)^{-1-a}, \quad C_{n} \sim a^{-1} n^{-2 / 3}(\log n)^{-1-a}$.

In this case conditions (5), (6), (7), (10) hold, and

$$
v(t) \sim \exp \left\{t^{-1 / a}\right\}, \quad t \rightarrow 0 .
$$

However, in this case condition (3) is not valid and Theorem 2 can not be applied.

2. If $A_{n} \sim c n^{1-\beta}$ for some $\beta>1$ and $c>0$, then condition $(13)$ is valid and the speed function

$$
v(t) \sim c^{-\frac{1}{\beta-1}} t^{\frac{1}{\beta-1}}, \quad t \rightarrow 0,
$$


suggests polynomial growth. This holds in particular, if $\lambda_{n}=\left(\begin{array}{c}2 n \\ 3\end{array}\right)$, with $\beta=2$. In this case, the process $2 Z(t)$ describes a triple-wise coalescent (in contrast to the pair-wise Kingman coalescent).

3. If $A_{n}=e^{-n^{\rho}}$ for some $\rho \in(0,1)$, then

$$
v(t) \sim\left(\log t^{-1}\right)^{1 / \rho}, \quad t \rightarrow 0
$$

In this case, both $(10)$ and $(9)$ are valid. Observe that for $\rho \in\left[\frac{1}{2}, 1\right)$, condition (12) holds for all $\epsilon \in(0,1)$ while condition (6) is not satisfied.

4. Turning to the Example 2 from Section 3.3 in [1], put $A_{n}=e^{-n / \log n}$. It was shown that in this case, $A_{n}^{-1} T_{n} \rightarrow 1$ in probability, but not almost surely. For this example, the speed function has the following asymptotics

$$
v(t) \sim\left(\log t^{-1}\right)\left(\log \log t^{-1}\right), \quad t \rightarrow 0 .
$$

Here, condition (9) is satisfied together with condition 12 , thus by Theorem 2 (ii), we have almost sure convergence $Z(t) / v(t) \rightarrow 1$.

5. If $A_{n}=e^{-n}$, then the fast decay of $A_{n}$ ensures that condition (4) is satisfied with $\alpha=1 / e$, and we have almost sure convergence $Z(t) / v(t) \rightarrow 1$ with

$$
v(t) \sim \log t^{-1}, \quad t \rightarrow 0 .
$$

For this example, condition (7) fails and the statements on the central limit theorem does not apply.

\section{Theorems on large deviations}

Consider a pure death processes whose rates regularly vary with index $\beta>1$ satisfying condition $(13)$. For a given $x>0$, define $\tau=\tau(x)$ as a solution of the equation

$$
\int_{1}^{\infty} \frac{d y}{(\beta-1)^{-1} y^{\beta}-\tau}=x .
$$

Observe that $\tau(x)$ is a strongly increasing function with

$$
\lim _{x \rightarrow 0} \tau(x)=-\infty, \quad \tau(1)=0, \quad \lim _{x \rightarrow \infty} \tau(x)=(\beta-1)^{-1} .
$$

Define two families of functions by

$$
I(x)=-(\beta-1) x \tau(x)-\ln (1-(\beta-1) \tau(x)),
$$

and $J(x)=x I\left(x^{\beta-1}\right)$, which are illustrated by Figure 1 Put

$$
c(\beta)=\left\{\frac{(1-1 / \beta) \pi}{\sin (\pi / \beta)}\right\}^{\beta /(\beta-1)}
$$



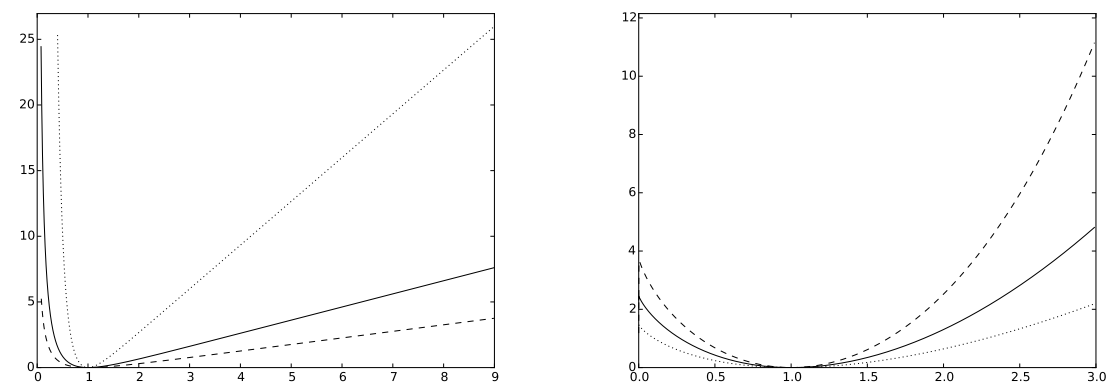

Figure 1: We use values $\beta=1.3$ (dotted lines), $\beta=2$ (solid lines), and $\beta=3$ (dashed lines) to present three pairs of profiles for the rate functions $I(x)$ on the left panel, and $J(x)$ on the right panel.

Lemma 4. The above defined functions $I(x)$ and $J(x)$ are both non-negative and strictly convex over $x \in(0, \infty)$ with $I(1)=J(1)=0$. They satisfy the following asymptotical relations

$$
\begin{aligned}
& I(x) \sim(\beta-1)^{-1} x, \quad x \rightarrow \infty, \\
& J(x) \sim(\beta-1)^{-1} x^{\beta}, \quad x \rightarrow \infty, \\
& I(x)=c(\beta) x^{-\frac{1}{\beta-1}}-\beta(\beta-1)^{-1} \ln x^{-1}-\ln c(\beta)-\beta+o(1), \quad x \rightarrow 0, \\
& J(x)=c(\beta)-(\beta \ln x+\ln c(\beta)+\beta) x+o(x), \quad x \rightarrow 0 .
\end{aligned}
$$

The next large deviation theorem extends a result derived in [4 for the Kingman coalescent.

Theorem 5. Consider a death process satisfying (13) with $\beta>1$.

(i) If $x \geq 1$, then

$$
\begin{aligned}
n^{-1} \log \mathbb{P}\left(T_{n}>x A_{n}\right) & \rightarrow-I(x), \quad n \rightarrow \infty, \\
v(t)^{-1} \log \mathbb{P}(Z(t)>x v(t)) & \rightarrow-J(x), \quad t \rightarrow 0 .
\end{aligned}
$$

(ii) If $0<x \leq 1$, then

$$
\begin{aligned}
n^{-1} \log \mathbb{P}\left(T_{n}<x A_{n}\right) & \rightarrow-I(x), \quad n \rightarrow \infty, \\
v(t)^{-1} \log \mathbb{P}(Z(t)<x v(t)) & \rightarrow-J(x), \quad t \rightarrow 0 .
\end{aligned}
$$

\section{Proof of Theorem 1}

We start with two lemmas. Lemma 6 is a version of the Kolmogorov inequality, needed in the proof of Lemma 7. Lemma 7 is used in the proof of Theorem 1 (iii) and Theorem 2 (ii). 
Lemma 6. If an infinite sum $\xi_{1}+\xi_{2}+\ldots$ of independent zero mean random variables converges almost surely, and $\zeta_{n}:=\xi_{n}+\xi_{n+1}+\ldots$, then for each $\epsilon>0$,

$$
\mathbb{P}\left(\sup _{k \geq n}\left|\zeta_{k}\right| \geq \epsilon\right) \leq \epsilon^{-2} \mathbb{E} \zeta_{n}^{2}, \quad n \geq 1 .
$$

Proof. It is easy to check that the sequence $\zeta_{n}$ forms a backward martingale. Putting $B_{k}=\left\{\left|\zeta_{k}\right| \geq \epsilon,\left|\zeta_{k+1}\right|<\epsilon,\left|\zeta_{k+2}\right|<\epsilon, \ldots\right\}$ and using the submartingale property of $\zeta_{n}^{2}$ we get

$$
\mathrm{E}\left(\zeta_{n}^{2}\right) \geq \sum_{k=n}^{\infty} \mathrm{E}\left(\zeta_{n}^{2} 1_{B_{k}}\right) \geq \sum_{k=n}^{\infty} \mathrm{E}\left(\zeta_{k}^{2} 1_{B_{k}}\right) \geq \epsilon^{2} \sum_{k=n}^{\infty} \mathrm{P}\left(B_{i}\right)=\epsilon^{2} \mathbb{P}\left(\sup _{k \geq n}\left|\zeta_{k}\right| \geq \epsilon\right) .
$$

Lemma 7. If 12 holds for some $\epsilon \in[0,1)$, then for any $\delta>0$,

$$
\mathbb{P}\left(\sup _{k \geq n} \frac{\left|T_{k}-A_{k}\right|}{A_{k(1-\epsilon)}}>\delta\right) \rightarrow 0, \quad n \rightarrow \infty .
$$

Proof. The following proof is an adaptation of the proof of Proposition 1 in [6]. For a given $n$, let $u_{n}$ be the unique natural number satisfying

$$
2^{-u_{n}-1}<A_{n(1-\epsilon)} \leq 2^{-u_{n}} .
$$

Clearly $u_{n} \leq u_{n+1}$ and $u_{n} \rightarrow \infty$. Putting $v_{j}=\min \left\{k: u_{k}=j\right\}$, we obtain

$$
\begin{aligned}
\mathbb{P}\left(\sup _{k \geq n} A_{k(1-\epsilon)}^{-1}\left|T_{k}-A_{k}\right|\right. & \geq \epsilon) \leq \sum_{j \geq u_{n}} \mathbb{P}\left(\max _{k: u_{k}=j} A_{k(1-\epsilon)}^{-1}\left|T_{k}-A_{k}\right| \geq \epsilon\right) \\
& \leq \sum_{j \geq u_{n}} \mathbb{P}\left(\max _{k: u_{k}=j}\left|T_{k}-A_{k}\right| \geq \epsilon 2^{-j-1}\right) \\
& \leq \sum_{j \geq u_{n}} \mathbb{P}\left(\sup _{k \geq v_{j}}\left|T_{k}-A_{k}\right| \geq \epsilon 2^{-j-1}\right) .
\end{aligned}
$$

Notice that for some $j$ the set of indices $\left\{k: u_{k}=j\right\}$ might be empty - in such a case the corresponding maximum is assumed to be zero.

Suppose condition $[12]$ holds for an $\epsilon \in[0,1)$. By Lemma 6 applied to

$$
\xi_{i}=X_{i}-\lambda_{i}^{-1}
$$

having centered exponential distributions, we see that there is a positive contant $c$ such that

$$
\begin{aligned}
\sum_{j \geq u_{n}} \mathbb{P}\left(\sup _{k \geq v_{j}}\left|T_{k}-A_{k}\right| \geq \epsilon 2^{-j-1}\right) & \leq \sum_{j \geq u_{n}} c \epsilon^{-2} 4^{j+1} \sum_{k \geq v_{j}} \lambda_{k+1}^{-2} \\
& =c \epsilon^{-2} \sum_{j \geq u_{n}} 4^{j+1} \sum_{l \geq j} 4^{-l} \sum_{k: u_{k}=l}\left(\lambda_{k+1} 2^{-l}\right)^{-2} \\
& \leq c \epsilon^{-2} \sum_{l \geq u_{n}} \sum_{j=u_{n}}^{l} 4^{j-l+1} \sum_{k: u_{k}=l}\left(\lambda_{k+1} A_{k(1-\epsilon)}\right)^{-2}
\end{aligned}
$$


Thus,

$$
\begin{aligned}
\mathbb{P}\left(\sup _{k \geq n} A_{k(1-\epsilon)}^{-1}\left|T_{k}-A_{k}\right|\right. & \leq 4 c \epsilon^{-2} \sum_{l \geq u_{n}} \sum_{k: u_{k}=l}\left(\lambda_{k+1} A_{k(1-\epsilon)}\right)^{-2} \\
& =4 c \epsilon^{-2} \sum_{k \geq K_{n}}\left(\lambda_{k+1} A_{k(1-\epsilon)}\right)^{-2}
\end{aligned}
$$

where $K_{n}=\min \left\{k: u_{k}=u_{n}\right\}$ is $v_{j}$ for $j=u_{n}$. By monotonicity of $A_{n}$, we have $K_{n} \rightarrow \infty$ as $n \rightarrow \infty$, and the statement of Lemma 7 follows.

Observe that for any given $u_{0}>0$, the moment generating function

$$
E e^{u T_{n}}=\prod_{i=n+1}^{\infty} \frac{\lambda_{i}}{\lambda_{i}-u}=\exp \left\{-\sum_{i=n+1}^{\infty} \log \left(1-u \lambda_{i}^{-1}\right)\right\}, \quad u \in\left(-\infty, u_{0}\right]
$$

is well-defined for all sufficiently large $n$.

Proof of Theorem 1 (I). By (15) and (8), we get for each $u \geq 0$,

$$
\mathbb{E} e^{-u T_{n} / A_{n}}=\prod_{k \geq n+1} \frac{1}{u\left(\lambda_{k} A_{n}\right)^{-1}+1} \rightarrow \prod_{i \geq 0} \frac{1}{u \alpha^{i}(1-\alpha)+1} .
$$

Proof of Theorem 1 (II). The stated convergence in probability is easily derived using the Chebyshev inequality, see Remark 4 in Section 2

Proof of Theorem 1 (III). The stated almost sure convergence is a straightforward corollary of Lemma 7 with $\epsilon=0$.

Proof of Theorem 1 (IV). Using (15) and notation (14), we find

$$
\mathbb{E} e^{u\left(\xi_{n}+\xi_{n+1}+\ldots\right)}=\exp \left\{-\sum_{i=n}^{\infty} u \lambda_{i}^{-1}+\log \left(1-t \lambda_{i}^{-1}\right)\right\} .
$$

Applying the Taylor formula for the logarithm we see that under condition (7),

$$
E e^{u B_{n}^{-1}\left(\xi_{n+1}+\xi_{n+2}+\ldots\right)} \sim \exp \left\{\sum_{i=n+1}^{\infty} \frac{\left(u B_{n}^{-1} \lambda_{i}^{-1}\right)^{2}}{2}\right\}=e^{u^{2} / 2} .
$$

\section{Proof of Theorem 2}

Observe that since

$$
\frac{Z\left(A_{n-1}\right)}{n} \leq \frac{Z(t)}{v(t)} \leq \frac{Z\left(A_{n}\right)}{n}, \quad n=v(t),
$$

convergence $\frac{Z(t)}{v(t)} \rightarrow 1$ as $t \rightarrow 0$ is equivalent to $\frac{Z\left(A_{n}\right)}{n} \rightarrow 1$ as $n \rightarrow \infty$. 
Proof of PART (I). Fix some arbitrary $\epsilon \in(0,1)$ and $u \in(0, \infty)$. Given (3), there exist a $\delta \in(0,1)$ and an $n_{0}=n_{0}(\epsilon, u)$ such that for all $n \geq n_{0}$,

$$
\begin{aligned}
& A_{n(1+\epsilon)} / A_{n}<\delta, \\
& A_{n} \lambda_{k}>2 \delta u, \quad k>n(1+\epsilon),
\end{aligned}
$$

and the moment generating function

$$
\mathbb{E} e^{u T_{n(1+\epsilon)} / A_{n}}=\prod_{k>n(1+\epsilon)} \frac{1}{1-\left(A_{n} \lambda_{k}\right)^{-1} u}
$$

is well defined. By Markov's inequality,

$$
\mathbb{P}\left(T_{n(1+\epsilon)}>A_{n}\right) \leq e^{-u} \mathbb{E} e^{u T_{n(1+\epsilon)} / A_{n}}=e^{-u} \exp \left\{-\sum_{k>n(1+\epsilon)} \ln \left(1-\left(A_{n} \lambda_{k}\right)^{-1}\right) u\right\},
$$

yielding

$$
\mathbb{P}\left(T_{n(1+\epsilon)}>A_{n}\right) \leq e^{-u} \exp \left\{\sum_{k>n(1+\epsilon)} \frac{\left(A_{n} \lambda_{k}\right)^{-1} u}{1-\left(A_{n} \lambda_{k}\right)^{-1} u}\right\} \leq e^{-u / 2} .
$$

Letting $u \rightarrow \infty$, we see that $\mathbb{P}\left(T_{n(1+\epsilon)}>A_{n}\right) \rightarrow 0$. Since

$$
\mathbb{P}\left(Z\left(A_{n}\right)>n(1+\epsilon)\right)=\mathbb{P}\left(T_{n(1+\epsilon)}>A_{n}\right),
$$

we conclude that $\mathbb{P}\left(Z\left(A_{n}\right)>n(1+\epsilon)\right) \rightarrow 0$. In the same way we can prove that $\mathbb{P}\left(Z\left(A_{n}\right)<n(1-\epsilon)\right) \rightarrow 0$ as $n \rightarrow \infty$.

ProOF OF PART (II). It suffices to prove that $Z\left(A_{n}\right) / n \rightarrow 1$ almost surely as $n \rightarrow \infty$ or, in other terms,

$$
\mathbb{P}\left(\sup _{k \geq n} \frac{Z\left(A_{k}\right)-k}{k}>\epsilon\right) \rightarrow 0, \quad \mathbb{P}\left(\inf _{k \geq n} \frac{Z\left(A_{k}\right)-k}{k}<-\epsilon\right) \rightarrow 0 .
$$

To check the first convergence, observe that

$$
\begin{aligned}
\mathbb{P}\left\{\sup _{k \geq n} \frac{Z\left(A_{k}\right)-k}{k}>\epsilon\right\} & =\mathbb{P}\left\{\exists k \geq n: Z\left(A_{k}\right)>(1+\epsilon) k\right\} \\
& =\mathbb{P}\left\{\exists k \geq n: T_{(1+\epsilon) k}>A_{k}\right\} \\
& =\mathbb{P}\left\{\exists k \geq n: \frac{T_{(1+\epsilon) k}-A_{(1+\epsilon) k}}{A_{k}}>1-\frac{A_{(1+\epsilon) k}}{A_{k}}\right\} .
\end{aligned}
$$

It follows that by condition (3), for some $\delta \in(0,1)$ and all $n \geq n_{0}(\epsilon)$,

$$
\begin{aligned}
\mathbb{P}\left\{\sup _{k \geq n} \frac{Z\left(A_{k}\right)-k}{k}>\epsilon\right\} & \leq \mathbb{P}\left\{\exists k \geq n: \frac{T_{(1+\epsilon) k}-A_{(1+\epsilon) k}}{A_{k}}>\delta\right\} \\
& \leq \mathbb{P}\left\{\exists k \geq n(1+\epsilon): \frac{T_{k}-A_{k}}{A_{k /(1+\epsilon)}}>\delta\right\},
\end{aligned}
$$


and it just remains to apply Lemma 7 The second convergence is verified similarly.

Proof of PART (III). The statement (iii) is an easy corollary of Theorem 1 (i) in view of Remark 2 in Section 2 and the relation

$$
\mathbb{P}\left(Z\left(A_{n}\right)>n+k\right)=\mathbb{P}\left\{\frac{T_{n+k}}{A_{n+k}}>\frac{A_{n}}{A_{n+k}}\right\} .
$$

ProOF OF PART (IV). The part (iv) immediately follows from Theorem 1 (iv) and equality

$$
\begin{aligned}
\mathbb{P}\left(\frac{Z\left(A_{n}\right)-n}{b(n)}>x\right) & =\mathbb{P}\left(T_{n+x b(n)}>A_{n}\right) \\
& =\mathbb{P}\left(\frac{T_{n+x b(n)}-A_{n+x b(n)}}{B_{n+x b(n)}}>\frac{A_{n}-A_{n+x b(n)}}{B_{n+x b(n)}}\right) .
\end{aligned}
$$

\section{Proof of Lemma 4}

Put

$$
\Lambda(u)=-\int_{1}^{\infty} \log \left(1-(\beta-1) u y^{-\beta}\right) d y, \quad u \leq 1 /(\beta-1),
$$

then $\tau(x)$ satisfies $\Lambda^{\prime}(\tau(x))=x$. This yields

$$
\tau^{\prime}(x)=1 / \Lambda^{\prime \prime}(\tau(x)) \text {. }
$$

Integration by parts gives

$$
\Lambda(\tau(x))=-\int_{1}^{\infty} \ln \left(1-(\beta-1) \tau(x) y^{-\beta}\right) d y=\ln (1-(\beta-1) \tau(x))+\beta x \tau(x) .
$$

Thus the defining expression for $I(x)$ can be rewritten as

$$
I(x)=x \tau(x)-\Lambda(\tau(x)), \quad x>0 .
$$

It follows that $I^{\prime}(x)=\tau(x)$ and $I^{\prime \prime}(x)=\tau^{\prime}(x)=1 / \Lambda^{\prime \prime}(\tau(x))$. In view of

$$
\Lambda^{\prime \prime}(u)=\int_{1}^{\infty} \frac{d y}{\left((\beta-1)^{-1} y^{\beta}-u\right)^{2}}>0,
$$

we conclude that $I(x)$ is a convex function with a minimal value $I(1)=0$.

On the other hand, $J(x)=x I\left(x^{\beta-1}\right)$ is also a convex function with minimal value $J(1)=0$. Indeed,

$$
J^{\prime}(x)=(\beta-1) x^{\beta-1} \tau\left(x^{\beta-1}\right)+I\left(x^{\beta-1}\right)=R\left(x^{\beta-1}\right),
$$

where $R(x)=(\beta-1) x \tau(x)+I(x)$. In particular, $J^{\prime}(1)=R(1)=0$. To verify that $R^{\prime}(x)>0$, observe that

$$
R^{\prime}(x)=(\beta-1) \tau(x)+(\beta-1) x \tau^{\prime}(x)+\tau(x)=\beta \tau(x)+(\beta-1) x \tau^{\prime}(x) .
$$


We have $R^{\prime}(x)=\tau^{\prime}(x) r(\tau(x))$, where $\tau^{\prime}(x)>0$ and

$$
\begin{aligned}
r(u) & =\beta u \Lambda^{\prime \prime}(u)+(\beta-1) \Lambda^{\prime}(u)=\int_{1}^{\infty} \frac{\beta u d y}{\left((\beta-1)^{-1} y^{\beta}-u\right)^{2}}+\int_{1}^{\infty} \frac{(\beta-1) d y}{(\beta-1)^{-1} y^{\beta}-u} \\
& =\int_{1}^{\infty} \frac{\left(y^{\beta}+u\right) d y}{\left((\beta-1)^{-1} y^{\beta}-u\right)^{2}} .
\end{aligned}
$$

Clearly, $r(u)>0$ for $u \geq-1$, and it remains to show that $r(-u)>0$ for $u>1$.

To see this, observe that in view of

$$
r(-u)=\int_{1}^{\infty} \frac{\left(y^{\beta}-u\right) d y}{\left((\beta-1)^{-1} y^{\beta}+u\right)^{2}}=u^{1 / \beta-1} \int_{u^{-1 / \beta}}^{\infty} \frac{\left(y^{\beta}-1\right) d y}{\left((\beta-1)^{-1} y^{\beta}+1\right)^{2}},
$$

we have

$$
r(-u)>u^{1 / \beta-1}(\beta-1)^{2} \int_{0}^{\infty} \frac{\left(y^{\beta}-1\right) d y}{\left(y^{\beta}+\beta-1\right)^{2}}=0,
$$

provided $\beta>1$.

Turning to the stated asymptotics as $x \rightarrow \infty$, put $h=1-(\beta-1) u, z=\frac{y^{\beta}-1}{h}$ and write

$$
\Lambda^{\prime \prime}(u)=\int_{1}^{\infty} \frac{d y}{\left((\beta-1)^{-1} y^{\beta}-u\right)^{2}}=\frac{(\beta-1)^{2}}{h^{2}} \int_{1}^{\infty} \frac{d y}{\left(\frac{y^{\beta}-1}{h}+1\right)^{2}}=\frac{(\beta-1)^{2}}{\beta h} \int_{0}^{\infty} \frac{(1+z h)^{1-1 / \beta}}{(z+1)^{2}} d z .
$$

This yields

$$
\Lambda^{\prime \prime}(u) \sim \frac{(\beta-1)^{2}}{\beta(1-(\beta-1) u)}, \quad u \rightarrow(1-\beta)^{-1} .
$$

Therefore, using L'Hospital's rule we find

$$
-x^{-1} \ln (1-(\beta-1) \tau(x)) \sim \frac{(\beta-1) \tau^{\prime}(x)}{1-(\beta-1) \tau(x)}=\frac{\beta-1}{\Lambda^{\prime \prime}(\tau(x))(1-(\beta-1) \tau(x))} \rightarrow \frac{\beta}{\beta-1}, \quad x \rightarrow \infty .
$$

This implies

$$
x^{-1} I(x)=-(\beta-1) \tau(x)-x^{-1} \ln (1-(\beta-1) \tau(x)) \rightarrow-1+\frac{\beta}{\beta-1}=\frac{1}{\beta-1} .
$$

The last assertion of the lemma gives an asymptotics as $x \rightarrow 0$. We prove it by first noticing that as $u \rightarrow \infty$,

$$
\begin{aligned}
\Lambda^{\prime}(-u) & =u^{1 / \beta-1} \int_{u^{-1 / \beta}}^{\infty} \frac{d y}{(\beta-1)^{-1} y^{\beta}+1} \\
& =u^{1 / \beta-1} \int_{0}^{\infty} \frac{d y}{(\beta-1)^{-1} y^{\beta}+1}-u^{1 / \beta-1} \int_{0}^{u^{-1 / \beta}} \frac{d y}{(\beta-1)^{-1} y^{\beta}+1} \\
& =u^{1 / \beta-1}(\beta-1)^{1 / \beta} \frac{\pi / \beta}{\sin (\pi / \beta)}-u^{-1}+o\left(u^{-1}\right),
\end{aligned}
$$


and therefore, as $x \rightarrow 0$,

$$
x=|\tau(x)|^{1 / \beta-1}(\beta-1)^{1 / \beta} \frac{\pi / \beta}{\sin (\pi / \beta)}-|\tau(x)|^{-1}+o\left(|\tau(x)|^{-1}\right) .
$$

Solving the last equation, we get as a first approximation

$$
\tau(x) \sim-b(\beta) x^{-\frac{\beta}{\beta-1}}, \quad b(\beta):=(\beta-1)^{\frac{1}{\beta-1}}\left(\frac{\pi / \beta}{\sin (\pi / \beta)}\right)^{\frac{\beta}{\beta-1}}=c(\beta) /(\beta-1),
$$

and then more exactly

$$
\tau(x)=-b(\beta) x^{-\frac{\beta}{\beta-1}}+x^{-1}+o\left(x^{-1}\right) .
$$

Thus

$I(x)=-(\beta-1) x \tau(x)-\ln (1-(\beta-1) \tau(x))=c(\beta) x^{-\frac{1}{\beta-1}}-\frac{\beta}{\beta-1} \ln x^{-1}-\ln c(\beta)-\beta+o(1)$.

and as stated

$$
J(x)=c(\beta)-(\beta \ln x+\ln c(\beta)+\beta) x+o(x) .
$$

\section{Proof of Theorem 5}

Here we prove only the statement (i), the statement (ii) is proved similarly. Our proof of (i) is more direct than that of [4 and uses the classical Cramer's device of 'tilted distributions'.

Let $x>1$. The required upper bound for (i) is obtained from

$$
\mathbb{P}\left(T_{n}>x A_{n}\right)=\mathbb{P}\left(e^{\tau(x) n A_{n}^{-1} T_{n}}>e^{x \tau(x) n}\right) \leq \mathbb{E} e^{\tau(x) n A_{n}^{-1} T_{n}} e^{-x \tau(x) n} .
$$

Indeed, using 15 we find

$$
n^{-1} \log \mathbb{P}\left(T_{n}>x A_{n}\right) \leq-\frac{1}{n} \sum_{i=n}^{\infty} \log \left(1-\frac{\tau(x)}{\lambda_{i} A_{n} n^{-1}}\right)-x \tau(x),
$$

and it remains to see that by the dominated convergence theorem,

$$
-\frac{1}{n} \sum_{i=n}^{\infty} \log \left(1-\frac{u}{\lambda_{i} A_{n} n^{-1}}\right)=\int_{1}^{\infty} \log \left(1-\frac{u}{\lambda_{y n} A_{n} n^{-1}}\right) d y \rightarrow \Lambda(u) .
$$

Here the dominating function is found from the uniform bounds

$$
(1-\epsilon) y^{\beta-\epsilon} \leq \frac{\lambda_{y n}}{\lambda_{n}} \leq(1+\epsilon) y^{\beta+\epsilon}, \quad n \geq n_{0}(\epsilon), \quad y \in[1, \infty),
$$

where $n_{0}(\epsilon)$ does not depend on $y$. 
The lower bound for (i) is derived using the so-called tilted distributions. For the random variables $X_{i}$ with exponential distributions $\operatorname{Exp}\left(\lambda_{i}\right)$ we introduce tilted versions $\tilde{X}_{i, n}$ with exponential distributions $\operatorname{Exp}\left(\tilde{\lambda}_{i, n}\right)$, where

$$
\tilde{\lambda}_{i, n}=\lambda_{i}-\tau(x) n A_{n}^{-1}, \quad i \geq n .
$$

The parameters $\left(\tilde{\lambda}_{i, n}\right)_{i \geq n}$ are positive for all sufficiently large $n$. Let $F_{n}(y)$ and $\tilde{F}_{n}(y)$ be the distributions functions for $T_{n}=\sum_{i=n+1}^{\infty} X_{i}$ and $\tilde{T}_{n}=\sum_{i=n+1}^{\infty} \tilde{X}_{i, n}$ respectively. We have

$$
\begin{aligned}
\int_{-\infty}^{\infty} e^{u y} d \widetilde{F}_{n}(y) & =\mathbb{E} e^{u \tilde{T}_{n}}=\prod_{i=n+1}^{\infty} \frac{\tilde{\lambda}_{i, n}}{\tilde{\tilde{\lambda}}_{i, n}-u} \\
& =\frac{\mathbb{E} e^{\left(u+\tau(x) n / A_{n}\right) T_{n}}}{\mathbb{E} e^{\left(\tau(x) n / A_{n}\right) T_{n}}}=\frac{1}{\mathbb{E} e^{\tau(x) n T_{n} / A_{n}}} \int_{-\infty}^{\infty} e^{\left(u+\tau(x) n / A_{n}\right) y} d F_{n}(y)
\end{aligned}
$$

implying

$$
d \widetilde{F}_{n}(y)=\frac{e^{\tau(x) n / A_{n} y}}{\mathbb{E} e^{\tau(x) n T_{n} / A_{n}}} d F_{n}(y) .
$$

Thus, for any $b>x$, we get

$$
\begin{aligned}
\mathbb{P}\left(T_{n}>x A_{n}\right) & =\int_{x A_{n}}^{\infty} d F_{n}(y) \geq \mathbb{E}\left[e^{\tau(x) n / A_{n} T_{n}}\right] e^{-\tau(x) n b} \int_{x A_{n}}^{b A_{n}} d \widetilde{F}_{n}(y) \\
& =\mathbb{E}\left[e^{\frac{\tau(x) n}{A_{n}} T_{n}}\right] e^{-\tau(x) n b} \int_{x A_{n}}^{b A_{n}} d \widetilde{F}_{n}(y) .
\end{aligned}
$$

By the dominated convergence theorem,

$$
\begin{aligned}
A_{n}^{-1} \mathbb{E} \widetilde{T}_{n} & =n^{-1} \sum_{i=n+1}^{\infty} \frac{1}{\lambda_{i} A_{n} n^{-1}-\tau(x)}=\int_{1}^{\infty} \frac{d y}{\lambda_{y(n+1)} A_{n} n^{-1}-\tau(x)} \\
& \rightarrow \int_{1}^{\infty} \frac{1}{y^{\beta}(\beta-1)^{-1}-\tau(x)} d y=\Lambda^{\prime}(\tau(x))=x .
\end{aligned}
$$

Similarly,

$$
\frac{n}{A_{n}^{2}} \widetilde{B}_{n}^{2} \rightarrow \int_{1}^{\infty} \frac{1}{\left(y^{\beta}(\beta-1)^{-1}-\tau(x)\right)^{2}} d y=\Lambda^{\prime \prime}(\tau(x))
$$

and

$$
\frac{n^{2}}{A_{n}^{3}} \widetilde{C}_{n}^{3} \rightarrow \int_{1}^{\infty} \frac{1}{\left(y^{\beta}(\beta-1)^{-1}-\tau(x)\right)^{3}} d y=\frac{1}{2} \Lambda^{\prime \prime \prime}(\tau(x)) .
$$

We see that $\tilde{C}_{n}=o\left(\tilde{B}_{n}\right)$ so that the distribution of $\frac{\sqrt{n}\left(\widetilde{T}_{n}-x A_{n}\right)}{A_{n}}$ is approximately normal with zero mean and variance $\Lambda^{\prime \prime}(\tau(x))$. Thus

$$
\int_{x A_{n}}^{b A_{n}} d \widetilde{F}^{n}(y) \rightarrow 1 / 2
$$


and we get

$$
\liminf _{n \rightarrow \infty} n^{-1} \log \mathbb{P}\left(T_{n}>x A_{n}\right) \geq \Lambda(\tau(x))-b \tau(x) .
$$

To finish the proof of the first part of (i) we send $b \rightarrow x$.

Turning to the second part of (i) it suffices to observe that as $n \rightarrow \infty$

$$
n^{-1} \log \mathbb{P}\left(Z\left(A_{n}\right)>n x\right) \sim x(n x)^{-1} \log \mathbb{P}\left(T_{n x}>x^{\beta-1} A_{n x}\right) \rightarrow x I\left(x^{\beta-1}\right) .
$$

\section{References}

[1] Bansaye, V., Méléard, S., and Richard, M.: Speed of coming down from infinity for birth and death processes, arXiv:1504.08160

[2] Berestycki, J., Berestycki, N., Limic, V.: The $\Lambda$-coalescent speed of coming down from infinity. Ann. Probab. 38 (2010) 207-233.

[3] Bingham, N. H., Goldie, C. M., and Teugels, J. L.: Regular Variation. Encyclopedia of mathematics and its Applications. Cambridge University Press, Cambridge, 1987.

[4] Depperschmidt, A., Pfaffelhuber, P., and Scheuringer, A.: Some large deviations in Kingman's coalescent. Electron. Commun. Probab. 20 (2015) $1-14$.

[5] Kingman, J. F. C.: The coalescent. Stochastic Process. Appl. 13 (1982) $235-248$.

[6] Klesov, O. I.: Rate of convergence of series of random variables. Ukrainian Math. Journal. 35 (1983) 309-314.

[7] Pakes, A. G.: Divergence rates for explosive birth processes. Stochastic Process. Appl. 41 (1992) 91-99.

[8] Sagitov, S.: On an explosive branching process. Theory Probab. Appl. 40 (1996) 575-577.

[9] Waugh, W. A. O'N.: Modes of Growth of Counting Processes with Increasing Arrival Rates. Journal Appl. Probab. 11 (1974) 237-247. 